\title{
Low-energy diets differing in fibre, red meat and coffee intake equally improve insulin sensitivity in type 2 diabetes: a randomised feasibility trial
}

\author{
Bettina Nowotny • Lejla Zahiragic • Alessandra Bierwagen • Stefan Kabisch • \\ Jan B. Groener • Peter J. Nowotny • Ann Kristin Fleitmann • Christian Herder • \\ Giovanni Pacini • Iris Erlund • Rikard Landberg • Hans-Ulrich Haering • \\ Andreas F. H. Pfeiffer • Peter P. Nawroth • Michael Roden
}

Received: 8 July 2014 / Accepted: 22 September 2014 / Published online: 26 November 2014

(C) Springer-Verlag Berlin Heidelberg 2014

\begin{abstract}
Aims/hypothesis Epidemiological studies have found that a diet high in fibre and coffee, but low in red meat, reduces the risk for type 2 diabetes. We tested the hypothesis that these
\end{abstract}

Bettina Nowotny and Lejla Zahiragic contributed equally to this manuscript.

Electronic supplementary material The online version of this article (doi:10.1007/s00125-014-3457-8) contains peer-reviewed but unedited supplementary material, which is available to authorised users.

B. Nowotny $\cdot$ L. Zahiragic $\cdot$ A. Bierwagen $\cdot$ P. J. Nowotny $\cdot$

A. K. Fleitmann $\cdot$ C. Herder $\cdot$ M. Roden $(\triangle)$

Institute for Clinical Diabetology, German Diabetes Center,

Leibniz Institute for Diabetes Research at Heinrich-Heine University,

Auf'm Hennekamp 65, D-40225 Düsseldorf, Germany

e-mail: michael.roden@ddz.uni-duesseldorf.de

B. Nowotny $\cdot$ L. Zahiragic $\cdot$ A. Bierwagen $\cdot$ P. J. Nowotny $\cdot$

A. K. Fleitmann $\cdot$ C. Herder $\cdot$ M. Roden

German Center for Diabetes Research, Partner Düsseldorf,

Düsseldorf, Germany

B. Nowotny $\cdot$ L. Zahiragic $\cdot$ M. Roden

Department of Endocrinology and Diabetology,

Heinrich-Heine University, Düsseldorf, Germany

S. Kabisch • A. F. H. Pfeiffer

Department Clinical Nutrition, German Institute

for Nutritional Research (DIfE) Potsdam,

Bergholz-Rehbrücke, Germany

S. Kabisch • A. F. H. Pfeiffer

Department of Endocrinology, Diabetes and Nutrition,

Charité Universitätsmedizin Berlin, Campus Benjamin Franklin,

Berlin, Germany

S. Kabisch • A. F. H. Pfeiffer

German Center for Diabetes Research, Partner Berlin,

Berlin, Germany nutritional modifications differentially improve whole-body insulin sensitivity (primary outcome) and secretion.

Methods Inclusion criteria were: age 18-69 years, BMI $\geq 30 \mathrm{~kg} / \mathrm{m}^{2}$, type 2 diabetes treated with diet, metformin or

J. B. Groener • P. P. Nawroth

Department for Internal Medicine I, University Clinics Heidelberg, Heidelberg, Germany

G. Pacini

Metabolic Unit, Institute of Biomedical Engineering, National

Research Council, Padua, Italy

I. Erlund

Department of Health and Functional Capacity, National Public

Health Institute, Helsinki, Finland

R. Landberg

Department of Food Science, Swedish University of Agricultural Science, Uppsala, Sweden

R. Landberg

Nutritional Epidemiology Unit, Institute for Environmental

Medicine, Karolinska Institutet, Stockholm, Sweden

H.-U. Haering

Department of Internal Medicine, Division of Endocrinology,

Diabetology, Vascular Medicine, Nephrology and Clinical Chemistry

and Institute of Diabetes Research and Metabolic Diseases,

University Hospital Tübingen, Tübingen, Germany

H.-U. Haering

German Center for Diabetes Research, Partner

Tübingen-Neuherberg, Tübingen, Germany 
acarbose and known disease duration of $\leq 5$ years. Exclusion criteria were: $\mathrm{HbA}_{1 \mathrm{c}}>75 \mathrm{mmol} / \mathrm{mol}(9.0 \%)$, type 1 or secondary diabetes types and acute or chronic diseases including cancer. Patients taking any medication affecting the immune system or insulin sensitivity, other than metformin, were also excluded. Of 59 patients (randomised using randomisation blocks [four or six patients] with consecutive numbers), 37 (54\% female) obese type 2 diabetic patients completed this controlled parallel-group 8-week low-energy dietary intervention. The participants consumed either a diet high in cereal fibre (whole grain wheat/rye: $30-50 \mathrm{~g} /$ day) and coffee ( $\geq 5$ cups/day), and free of red meat (L-RISK, $n=17$ ) or a diet low in fibre ( $\leq 10 \mathrm{~g} /$ day $)$, coffee-free and high in red meat $(\geq 150 \mathrm{~g} /$ day) diet (H-RISK, $n=20)$. Insulin sensitivity and secretion were assessed by hyperinsulinaemic-euglycaemic clamp and intravenous glucose tolerance tests with isotope dilution. Whole-body and organ fat contents were measured by magnetic resonance imaging and spectroscopy.

Results Whole-body insulin sensitivity increased in both groups (mean [95\% CI]) (H-RISK vs L-RISK: $0.8[0.2,1.4]$ vs $\left.1.0[0.4,1.7] \mathrm{mg} \mathrm{kg}^{-1} \mathrm{~min}^{-1}, p=0.59\right)$, while body weight decreased $(-4.8 \%[-6.1 \%,-3.5 \%]$ vs $-4.6 \%[-6.0 \%,-3.3 \%]$, respectively). Hepatic insulin sensitivity remained unchanged, whereas hepatocellular lipid content fell in both groups $(-7.0 \%[-9.6 \%,-4.5 \%]$ vs $-6.7 \%[-9.5 \%,-3.9 \%])$. Subcutaneous fat mass $\left(-1,553[-2,767,-340] \mathrm{cm}^{3}\right.$ vs -751 $[-2,047 ; 546] \mathrm{cm}^{3}$, respectively) visceral fat mass $(-206$ $[-783,371] \mathrm{cm}^{3}$ vs $-241[-856,373] \mathrm{cm}^{3}$, respectively) and muscle fat content $(-0.09 \%[-0.16 \%,-0.02 \%]$ vs $-0.02 \%$ $[-0.10 \%, 0.05 \%]$, respectively) decreased similarly. Insulin secretion remained unchanged, while the proinflammatory marker IL-18 decreased only after the L-RISK diet.

Conclusions/interpretation No evidence of a difference between both low-energy diets was identified. Thus, energy restriction per se seems to be key for improving insulin action in phases of active weight loss in obese type 2 diabetic patients, with a potential improvement of subclinical inflammation with the L-RISK diet.

Trial Registration: Clinicaltrials.gov NCT01409330

Funding: This study was supported by the Ministry of Science and Research of the State of North Rhine-Westphalia (MIWF NRW), the German Federal Ministry of Health (BMG), the Federal Ministry for Research (BMBF) to the Center for Diabetes Research (DZD e.V.) and the Helmholtz Alliance Imaging and Curing Environmental Metabolic Diseases (ICEMED).

Keywords Diet · Inflammation · Insulin sensitivity · Type 2 diabetes $\cdot$ Weight loss
Abbreviations
AIR Acute insulin response
BCAA Branched chain amino acids

\begin{tabular}{|c|c|}
\hline DHBA & 3,5-Dihydroxybenzoic acid \\
\hline DHPPA & 3-(3,5-Dihydroxyphenyl)-propanoic acid \\
\hline DI & Disposition index \\
\hline EGP & Endogenous glucose production \\
\hline FA & Fatty acids \\
\hline GDRS & German Diabetes Risk Score \\
\hline HCL & Hepatocellular lipid content \\
\hline H-RISK & $\begin{array}{l}\text { Higher-risk diet: low fibre coffee-free, } \\
\text { high red meat diet }\end{array}$ \\
\hline IMCL & Intramyocellular lipids \\
\hline IQR & Interquartile range \\
\hline jMRUI & $\begin{array}{l}\text { Java-based Magnetic Resonance } \\
\text { User Interface }\end{array}$ \\
\hline L-RISK & $\begin{array}{l}\text { Low-risk diet: high whole grain fibre } \\
\text { and coffee, red meat-free diet }\end{array}$ \\
\hline $\mathrm{PKC} \theta$ & Protein kinase- $\mathrm{C}$ theta \\
\hline$R_{\mathrm{d}}$ & Glucose disappearance \\
\hline SCAT & Truncal subcutaneous adipose tissue \\
\hline $\mathrm{TE}$ & Echo time \\
\hline TE eff & Effective echo time \\
\hline TR & Repetition time \\
\hline VAT & Visceral adipose tissue \\
\hline$\dot{V} \mathrm{CO}_{2}$ & Carbon dioxide production \\
\hline$\dot{V} \mathrm{O}_{2}$ & Oxygen consumption \\
\hline
\end{tabular}

\section{Introduction}

Several clinical scores predicting the future risk of type 2 diabetes have been developed comprising classical risk factors such as age, sex and BMI, in addition to other parameters [1-3]. The Finnish diabetes risk score integrates dietary components such as fibre [1], while the German Diabetes Risk Score (GDRS) also includes coffee and red meat consumption [2]. Results of observational studies support the protective effects of high fibre and coffee intake and reduced red meat consumption [4-6], but randomised intervention trials are needed to exclude unrecognised confounders and to reduce diabetes-related mortality in the future [7].

Few randomised controlled trials have tested the effects of dietary components on key mechanisms underlying the pathogenesis of type 2 diabetes, such as impaired insulin sensitivity and secretion. Isoenergetic diets with higher fibre intake improved insulin sensitivity in overweight glucose-tolerant humans [8], while no change in insulin sensitivity was observed in other studies comparing whole grain with refined grain intake [9]. Regular coffee drinking did not affect glucose homeostasis despite its anti-inflammatory effects [10], while a single cup of coffee even worsened postprandial glucose excursion in individuals with and without type 2 diabetes [11]. This may have resulted from adrenaline 
(epinephrine) release and adenosine receptor inhibition followed by impaired insulin sensitivity [11].

Greater consumption of red or processed meat could decrease insulin sensitivity because of its high protein and fat content, both of which can inhibit insulin action via mTOR, toxic lipid intermediates, inflammatory pathways or oxidative stress [12-14], and elevated branched chain amino acids (BCAA) are predictors of incident type 2 diabetes [15]. On the other hand, both amino acids and fatty acids (FA) can enhance insulin secretion and thereby counteract a reduction in insulin sensitivity $[16,17]$. At present, no controlled trials have reported the effects of red meat consumption on insulin sensitivity and glucose metabolism.

We hypothesised that a dietary intervention combining the nutrients that best predict a lower diabetes risk according to the GDRS would improve insulin sensitivity and subclinical inflammation beyond the effects of weight loss in short-term, well-controlled type 2 diabetes. Thus, this randomised controlled parallel group feasibility trial examines the effects of a moderate energy-restricted diet, as recommended by the ADA, with either increased fibre and coffee consumption and a lack of red meat (low-risk diet, L-RISK) or with increased red meat consumption, low in fibre and lacking coffee (higher-risk diet, H-RISK) on whole-body insulin sensitivity (primary endpoint), insulin secretion, body and tissue fat content and markers of inflammation.

\section{Methods}

Volunteers All participants gave written informed consent before their inclusion in the study (Clinicaltrials.gov registration number: NCT01409330), which was performed according to the Declaration of Helsinki (2008 version) and approved by the local ethics board. Inclusion criteria were: age 18-69 years, $\mathrm{BMI} \geq 30 \mathrm{~kg} / \mathrm{m}^{2}$, type 2 diabetes treated with diet, metformin or acarbose and known disease duration of $\leq 5$ years. Exclusion criteria were: $\mathrm{HbA}_{1 \mathrm{c}}>75 \mathrm{mmol} / \mathrm{mol}(9.0 \%)$, type 1 or secondary diabetes types and acute or chronic diseases including cancer. Patients taking any medication affecting the immune system or insulin sensitivity, other than metformin, were also excluded. Screening included medical history, laboratory tests, anthropometry and 12-lead ECG. All participants were randomly allocated to one intervention at one centre using randomisation blocks (four or six patients) with consecutive numbers. Patients were instructed to maintain their medication and physical activity assessed by weekly protocols.

Experimental design After randomisation, participants maintained their dietary habits during the run-in period $(19 \pm$ 8 days), and were monitored by daily dietary weighing protocols to calculate average energy intake (Prodi expert 6.1, Nutri-Science, Hausach, Germany). Metformin was stopped 3 days before and during the experimental tests, which were performed on 3 days within a single week [18]. After an overnight fast of 10-12 h, the participants reported to one of the three clinical research centres (Düsseldorf, BerlinPotsdam, Heidelberg) for metabolic tests. During the intervention, all participants received individually calculated daily diet sheets providing a $1,256 \mathrm{~kJ}$ reduction in total daily energy intake and a constant macronutrient distribution $(50 \%$ of energy from carbohydrates, $30 \%$ from fat, $20 \%$ from protein). The L-RISK diet contained 30-50 g/day cereal fibre from wheat and rye (100 g of wholegrain crispbread and 250 $300 \mathrm{~g}$ of wheat/rye wholegrain bread), $\geq 5$ cups of coffee/day containing 7-8 g coffee powder each and no red meat (although poultry was allowed). The H-RISK diet contained $\leq 10 \mathrm{~g}$ /day whole grain fibre, $\geq 150 \mathrm{~g}$ /day red meat (beef) and no coffee or tea. Patients were asked to document any changes and return the completed sheet to monitor compliance. The weekly visits also included monitoring of body weight, hip and waist circumference, blood pressure and blood sampling. After 8 weeks of intervention, the participants again stopped metformin for 3 days and underwent tests identical to those at baseline.

Modified Botnia clamp After fasting blood sampling, participants received a primed $(0.36 \mathrm{mg}$ [fasting blood glucose in $\mathrm{mmol} / \mathrm{l}] \times[5 \mathrm{mmol} / \mathrm{l}]^{-1} \times[\text { body weight in } \mathrm{kg}]^{-1} \mathrm{~min}^{-1}$ for $5 \mathrm{~min})$, continuous intravenous infusion $(0.036 \mathrm{mg} \times$ [body weight in $\left.\mathrm{kg}]^{-1} \mathrm{~min}^{-1}\right)$ of $\left[6,6-{ }^{2} \mathrm{H}_{2}\right]$ glucose $\left({ }^{2} \mathrm{H}\right.$ glucose, $99 \%$ enriched; CIL, Andover, MA, USA) started at $-120 \mathrm{~min}$ to assess endogenous glucose production (EGP) [18]. At $0 \mathrm{~min}$, an intravenous glucose bolus $(1 \mathrm{mg} / \mathrm{kg}$ body weight in a $30 \%$ [wt/vol.] solution containing $1.98 \%$ of $\left[6,6-{ }^{2} \mathrm{H}_{2}\right]$ glucose) was injected within $60 \mathrm{~s}$ and blood samples were obtained every $2 \mathrm{~min}$ for the first $10 \mathrm{~min}$, and then every $10 \mathrm{~min}$ up to $1 \mathrm{~h}$ to assess glucose-induced insulin secretion. The hyperinsulinaemic-euglycaemic clamp was then performed for $3 \mathrm{~h}$ using a primed-continuous insulin infusion $(10 \mathrm{mU}$ [body weight in $\mathrm{kg}]^{-1} \mathrm{~min}^{-1}$ for $10 \mathrm{~min}$, followed by $1.5 \mathrm{mU}$ [body weight in $\mathrm{kg}]^{-1} \min ^{-1}$; Insuman Rapid, sanofi-aventis, Frankfurt am Main, Germany) to assess insulin sensitivity. A variable infusion of $20 \%$ glucose (wt/vol.; B. Braun, Melsungen, Germany) enriched with $2 \%\left[6,6-{ }^{2} \mathrm{H}_{2}\right]$ glucose (wt/vol.) was administered to maintain blood glucose at $5 \mathrm{mmol} / \mathrm{l}$ [18]. Plasma glucose was measured at $5 \mathrm{~min}$ intervals.

Indirect calorimetry Indirect calorimetry was performed in the canopy mode (Vmax Encore 29n, CareFusion, Höchberg, Germany) during fasting and steady-state clamp conditions for $20 \mathrm{~min}$ followed by a post-exposure recalibration procedure [19]. Oxygen consumption $\left(\dot{V} \mathrm{O}_{2}\right)$ and carbon dioxide production $\left(\dot{V} \mathrm{CO}_{2}\right)$ were measured and substrate oxidation rates were calculated as previously described [13]. Non-oxidative glucose disposal was 
calculated as the difference between glucose disappearance $\left(R_{\mathrm{d}}\right)$ and carbohydrate oxidation.

Laboratory analyses Blood samples were immediately chilled and centrifuged, and supernatants stored at $-20^{\circ} \mathrm{C}$ until analysis. Whole-blood glucose concentration was measured at the bedside (EKF biosen C-Line glucose analyser, EKF Diagnostics, Barleben, Germany) [20]. Serum triacylglycerols, cholesterol and liver enzymes were analysed using a Cobas c311 analyser (Roche Diagnostics, Mannheim, Germany). FA, C-peptide, insulin, glucagon, glucagon-like peptide 1 (GLP-1) and gastric inhibitory peptide (GIP) were measured as previously described [13]. Serum cytokines were assayed using the Quantikine HS (IL-6) and Quantikine (total adiponectin, IL-1RA) ELISA kits (R\&D Systems, Wiesbaden, Germany) and an IL-18 ELISA kit (MBL, Nagoya, Japan) [21]. Serum amino acids were measured by GC-MS using an EZ:faast kit (Phenomenex, Torrance, CA, USA) with Norvaline as internal standard. Plasma coffeederived compounds (caffeine and its metabolites, theophylline, theobromine and paraxanthine, and the polyphenols caffeic acid and its metabolites, dihydrocaffeic acid, ferulic acid, dihydroferulic acid, isoferulic acid, dihydroisoferulic acid, dihydro-3-coumaric acid, 3-(3,4-dimethoxyphenyl)propionic acid, 3,4-dimethylcaffeic acid and 3-coumaric acid) were determined using HPLC and GC-MS [10]. The two main urinary alkylresorcinol metabolites 3,5-dihydroxybenzoic acid (DHBA) and 3-(3,5-dihydroxyphenyl)-propanoic acid (DHPPA) were analysed using GC-MS [22]. Blood atom per cent enrichment of ${ }^{2} \mathrm{H}$ was measured by GC-MS (Agilent 6890 and 5975, Santa Clara, CA, USA) as previously described [13].

Magnetic resonance imaging and spectroscopy The experiments were performed on a clinical $3 \mathrm{~T}$ (Philips X-series Achieva, Best, the Netherlands) or a $1.5 \mathrm{~T}$ (Magnetom, Siemens Healthcare, Erlangen, Germany) whole-body magnet using standardised procedures [23].

Hepatocellular lipid content (HCL) was quantified from non-water and water-suppressed ${ }^{1} \mathrm{H}$ MR spectra using stimulated echo acquisition mode (STEAM) and absolute concentrations were expressed as per cent hepatocellular lipids relative to water content [24]. Truncal subcutaneous (SCAT) and visceral (VAT) adipose tissue were measured by whole-body imaging using transverse multislice turbo spin echo (TSE) sequences (repetition time/effective echo time: $\mathrm{TR} / \mathrm{TE}_{\mathrm{eff}}=$ $400-510 / 38 \mathrm{~ms}$ for $3 \mathrm{~T}$ and $\mathrm{TR} / \mathrm{TE}=490 / 12 \mathrm{~ms}$ for $1.5 \mathrm{~T}$ ) with a turbo factor of 7 and using the quadrature body coil [25].

Intramyocellular lipids (IMCL) were measured in the left calf using FLEX-S coils (Philips Healthcare, Best, the Netherlands) and a single-voxel point-resolved spectroscopy (PRESS) sequence $(\mathrm{TR} / \mathrm{TE}=2,000 / 32 \mathrm{~ms}$ ) with a voxel size of
$2 \mathrm{~cm}$ placed within the soleus and tibialis anterior muscles. ${ }^{1} \mathrm{H}$ MR spectra were processed using Java-based Magnetic Resonance User Interface (jMRUI; www.mrui.uab.es/mrui/; version 4.0) and IMCL quantified from the peak areas of the signal from middle chain methylene groups $(-\mathrm{CH} 2-$, at $1.3 \mathrm{ppm}$ ) relative to water signals in the same voxel.

Calculations and statistical analyses EGP before the start of the clamp ( $\min -30$ to 0 ), whole-body insulin sensitivity $\left(R_{\mathrm{d}}\right.$, primary outcome) and hepatic insulin sensitivity (per cent EGP suppression by insulin) were determined from the tracer infusion rate of D-[6,6- $\left.{ }^{2} \mathrm{H}_{2}\right]$ glucose and its enrichment of hydrogen bound to carbon 6 divided by the mean per cent enrichment of plasma D-[6,6- $\left.{ }^{2} \mathrm{H}_{2}\right]$ glucose [13]. The acute insulin response (AIR; pmol/1), as a measure of first phase insulin secretion during IVGTT, and the disposition index (DI), an index of beta cell function corrected for insulin sensitivity, were calculated as described previously [26]. For the feasibility study, change in whole-body insulin sensitivity was the primary outcome to obtain effect size and variance estimates for both diets; all other analyses have been predefined secondary exploratory analyses carried out on potential mechanisms.

Normally distributed parameters are presented as means \pm $\mathrm{SD}$, otherwise as median (interquartile range [IQR]). Notnormally distributed data were $\log _{e}$-transformed to achieve near-normal distribution and compared using a two-tailed Student's $t$ test for unpaired samples for comparisons between groups and paired analysis (before and after intervention). Mean change within-group is given with $95 \% \mathrm{CI}$, and the difference of the within-group changes were compared using general linear models with estimating least square means and 95\% CI adjusted for the respective baseline value. Pearson correlation coefficients were calculated. Statistical significance of differences was set at $p<0.05$. Calculations were performed using GraphPad Prism version 6.03 (GraphPad Software, La Jolla, CA, USA) and SAS Version 9.2 TS Level 2M3 (SAS Institute, Cary, NC, USA).

\section{Results}

Study population Between April 2011 and October 2013, 216 patients were contacted. Of these, 90 did not meet the inclusion criteria, 33 declined to participate and 34 were excluded for other reasons. Of the remaining 59 randomised participants (30 in the L-RISK group, 29 in the H-RISK group), 22 patients (13 in L-RISK, nine in H-RISK) discontinued the study during the run-in or treatment phase or were excluded from the analysis (electronic supplementary material [ESM] Fig. 1). The groups did not differ in baseline characteristics (Table 1). 
Macronutrient intake At baseline, total energy and macronutrient intake was comparable between groups, although red and white meat intake was higher in the L-RISK group (Table 1). During the intervention period, the average total energy intake decreased similarly in both groups (Table 2).

Table 1 Patient characteristics

\begin{tabular}{|c|c|c|}
\hline Characteristic & H-RISK & L-RISK \\
\hline$n$ (male/female) & $20(9 / 11)$ & $17(8 / 9)$ \\
\hline Age (years) & $53 \pm 10$ & $55 \pm 7$ \\
\hline Weight (kg) & $103 \pm 16$ & $106 \pm 19$ \\
\hline BMI $\left(\mathrm{kg} / \mathrm{m}^{2}\right)$ & $34.1(31.8,36.9)$ & $35.4(33.1,38.2)$ \\
\hline Waist circumference $(\mathrm{cm})$ & $112 \pm 11$ & $114 \pm 12$ \\
\hline Hip circumference (cm) & $113 \pm 14$ & $117 \pm 11$ \\
\hline Systolic BP (mmHg) & $135 \pm 17$ & $135 \pm 14$ \\
\hline Diastolic BP (mmHg) & $87 \pm 14$ & $85 \pm 10$ \\
\hline Triacylglycerols (mmol/l) & $1.50(1.12,2.79)$ & $1.55(1.35,2.16)$ \\
\hline Total cholesterol (mmol/l) & $5.56(4.94,6.13)$ & $5.28(4.80,5.80)$ \\
\hline LDL-cholesterol (mmol/l) & $3.45(2.78,3.99)$ & $3.34(2.80,3.79)$ \\
\hline HDL-cholesterol (mmol/l) & $1.18(1.06,1.42)$ & $1.22(1.10,1.40)$ \\
\hline $\operatorname{ALT}(\mu \mathrm{kat} / \mathrm{l})$ & $0.58(0.36,0.79)$ & $0.50(0.41,0.68)$ \\
\hline AST $(\mu \mathrm{kat} / \mathrm{l})$ & $0.43(0.33,0.54)$ & $0.37(0.29,0.46)$ \\
\hline Fasting glucose (mmol/l) & $6.7(5.5,7.6)$ & $5.9(5.3,6.8)$ \\
\hline Fasting insulin (pmol/l) & $\begin{array}{l}104.4(80.4 \\
147.1)\end{array}$ & $\begin{array}{c}90.1(72.5 \\
152.9)\end{array}$ \\
\hline $\mathrm{HbA}_{1 \mathrm{c}}(\mathrm{mmol} / \mathrm{mol})$ & $45(41,52)$ & $48(45,52)$ \\
\hline $\mathrm{HbA}_{1 \mathrm{c}}(\%)$ & $6.3(5.9,6.9)$ & $6.5(6.3,6.9)$ \\
\hline Diabetes duration (years) & $0.8(0.5,2.1)$ & $1.9(0.7,3.2)$ \\
\hline Metformin treatment (yes/no) ${ }^{\mathrm{a}}$ & $16 / 4$ & $13 / 4$ \\
\hline Total energy intake (kJ/day) & $8,881 \pm 1,778$ & $9,402 \pm 2,084$ \\
\hline Protein intake ( $\%$ of energy) & $17.3 \pm 3.8$ & $19.3 \pm 5.2$ \\
\hline $\begin{array}{l}\text { Carbohydrate intake (\% of } \\
\text { energy) }\end{array}$ & $45.3 \pm 7.3$ & $45.9 \pm 6.8$ \\
\hline Fat intake ( $\%$ of energy) & $39.5 \pm 7.6$ & $36.9 \pm 8.5$ \\
\hline Cereal fibre intake (g/day) & $8.7(6.5,11.9)$ & $9.2(7.6,13.0)$ \\
\hline Red meat intake (g/day) & $77(57,134)$ & $109(70,160)$ \\
\hline White meat intake (g/day) & $19(6,41)$ & $53(34,80)$ \\
\hline Plasma caffeine $(\mu \mathrm{mol} / \mathrm{l})$ & $3.0(1.5,4.0)$ & $3.4(2.1,4.3)$ \\
\hline $\begin{array}{c}24 \text { h urinary AR metabolite } \\
\text { excretion }(\mu \mathrm{mol} / 24 \mathrm{~h})\end{array}$ & $27.8(14.8,48.4)$ & $28.8(19.3,53.2)$ \\
\hline Total serum amino acids (mmol/l) & $2.71 \pm 0.50$ & $2.80 \pm 0.43$ \\
\hline $\mathrm{BCAA}(\mu \mathrm{mol} / \mathrm{l})$ & $494 \pm 142$ & $518 \pm 100$ \\
\hline Histidine $(\mu \mathrm{mol} / \mathrm{l})$ & $42 \pm 11$ & $48 \pm 16$ \\
\hline IL-6 (pg/ml) & $2.40(1.92,3.32)$ & $2.70(2.13,3.94)$ \\
\hline IL-18 (pg/ml) & $300(216,380)$ & $248(192,301)$ \\
\hline Adiponectin $(\mu \mathrm{g} / \mathrm{ml})$ & $3.64(2.49,5.42)$ & $4.85(3.79,7.56)$ \\
\hline IL-1RA (pg/ml) & $594(390,863)$ & $717(413,1,197)$ \\
\hline
\end{tabular}

Values given are means $\pm \mathrm{SD}$ or median (IQR)

${ }^{a}$ No patient received any glucose-lowering treatment other than metformin

ALT, alanine aminotransferase; AR, alkylresorcinol; AST, aspartate aminotransferase
Carbohydrate intake increased in both groups, while the decrease in fat intake and increase in protein intake was more pronounced in the L-RISK compared with the H-RISK group $(p<0.05)$. Cereal fibre intake from wheat and rye was comparable at baseline (Table 1) and increased by $19.8 \pm 10.0 \mathrm{~g} /$ day in the L-RISK group, but decreased by $5.0 \pm 4.7 \mathrm{~g} /$ day in the H-RISK group ( $p<0.0001$ vs L-RISK) during the intervention (Fig. 1a). Plasma caffeine concentration increased in the L-RISK group by $2.7 \pm 2.9 \mu \mathrm{mol} / \mathrm{l}$ and decreased in the H-RISK group by $2.5 \pm 2.2 \mu \mathrm{mol} / \mathrm{l}(p<0.0001$ vs L-RISK; Fig. $1 \mathrm{~b})$. The $24 \mathrm{~h}$ urinary excretion of DHBA and DHPPA (biomarkers of whole grain wheat and rye intake) slightly decreased by $10.7 \pm$ $12.9 \mu \mathrm{mol} / 24 \mathrm{~h}$ in the H-RISK group and increased by $60.7 \pm$ $59.7 \mu \mathrm{mol} / 24 \mathrm{~h}$ in the L-RISK group ( $p<0.0001$ vs H-RISK; Fig. 1c). Red meat intake decreased in the L-RISK group to $0 \mathrm{~g} /$ day, consistent with the protocol, but increased in the H-RISK group by $99 \pm 40 \mathrm{~g} /$ day ( $p<0.0001$ vs L-RISK; Fig. 1d). Intake of white meat was lower in the H-RISK group at baseline (Table 1) and decreased to $0 \mathrm{~g} /$ day, while it increased in the L-RISK group by $72 \pm 50 \mathrm{~g} /$ day ( $p<0.0001$ vs H-RISK; Fig. $1 \mathrm{~d}$ ). Total serum amino acid and BCAA concentrations did not change during the intervention, but histidine increased in the H-RISK group ( $p<0.001$ vs L-RISK; Table 2$)$.

Body weight and body composition In line with the study protocol, the relative reduction in body weight during the run-in period was $<5 \%$ of screening weight in all patients. During the intervention period, the average body weight reduction (mean $[95 \% \mathrm{CI}]$ ) was $-4.8 \%(-6.1 \%,-3.5 \%)$ in the H-RISK group and $-4.6 \%[-6.0 \%,-3.3 \%]$ in the L-RISK group when compared with the run-in period (Fig. 2a) with no difference between groups $(p=0.87)$. Waist circumference decreased in both groups $(p=0.17$, H-RISK vs L-RISK; Fig. 2b). In addition, the reduction of HCL in the H-RISK $(-7.0 \%[-9.6 \%,-4.5 \%])$ and L-RISK $(-6.7 \%$ [ $-9.5 \%$, $-3.9 \%]$ ) groups was similar (mean difference $-0.3 \%$ $[-4.1 \%, 3.5 \%], p=0.87$; Fig. $2 \mathrm{c}$ ). We observed no differences between H-RISK and L-RISK groups for a change in SCAT $\left(-1,553[-2,767,-340] \mathrm{cm}^{3}\right.$ vs $\left.-751[-2,047,546] \mathrm{cm}^{3}\right)$, VAT $\left(-206[-783,371] \mathrm{cm}^{3}\right.$ vs $\left.-241[-856,373] \mathrm{cm}^{3}\right)$ and IMCL $(-0.09 \%[-0.16 \%,-0.02 \%]$ vs $-0.02 \%[-0.10 \%, 0.05 \%])$, although the within-group reduction in the H-RISK group was more pronounced for SCAT and IMCL in soleus muscle (Fig. 2d-f).

Tissue-specific insulin sensitivity $R_{\mathrm{d}}$ was comparable at baseline in the H-RISK and L-RISK groups $(5.6 \pm 0.5$ vs $5.8 \pm$ $0.4 \mathrm{mg} \mathrm{kg}^{-1} \mathrm{~min}^{-1}, p=0.91$ ) and improved similarly (H-RISK vs L-RISK: $0.8[0.2,1.4]$ vs $1.0[0.4,1.7] \mathrm{mg} \mathrm{kg}^{-1} \mathrm{~min}^{-1}$; mean difference $-0.2[-1.1,0.6] \mathrm{mg} \mathrm{kg}^{-1} \min ^{-1}, p=0.59$; Fig. 3a). Non-oxidative glucose utilisation increased in the H-RISK compared with the L-RISK group (mean difference 1.18 $\left.[0.31,2.05] \mathrm{mg} \mathrm{kg}^{-1} \mathrm{~min}^{-1}, p=0.01\right)$, while glucose oxidation 
Table 2 Changes during intervention period

\begin{tabular}{|c|c|c|c|}
\hline & H-RISK ${ }^{\mathrm{a}}$ & L-RISK $^{\mathrm{a}}$ & Mean difference ${ }^{b}$ \\
\hline Total energy intake (kJ/day) & $-1,558(-1,849,-1,268)$ & $-1,194(-1,509,-879)$ & $-365(-795,65)$ \\
\hline Fat intake from energy $(\%)$ & $-6.7(-7.9,-5.5)$ & $-8.8(-10.2,-7.5)$ & $2.1(0.3,4.0)^{*}$ \\
\hline Carbohydrate intake from energy (\%) & $4.1(2.5,5.7)$ & $5.7(3.9,7.4)$ & $-1.5(-3.9,0.9)$ \\
\hline Protein intake from energy $(\%)$ & $2.5(2.1,2.9)$ & $3.1(2.7,3.6)$ & $-0.7(-1.3,-0.1)^{*}$ \\
\hline Triacylglycerols $(\mathrm{mmol} / \mathrm{l})^{\mathrm{c}}$ & $-0.22(-0.40,-0.03)$ & $-0.26(-0.47,-0.06)$ & $0.05(-0.23,0.32)$ \\
\hline Total cholesterol $(\mathrm{mmol} / \mathrm{l})^{\mathrm{c}}$ & $-0.04(-0.09,0.02)$ & $-0.08(-0.14,-0.02)$ & $0.04(-0.04,0.13)$ \\
\hline LDL-cholesterol $(\mathrm{mmol} / \mathrm{l})^{\mathrm{c}}$ & $-0.001(-0.068,0.067)$ & $-0.084(-0.161,-0.007)$ & $0.083(-0.019,0.186)$ \\
\hline HDL-cholesterol $(\mathrm{mmol} / \mathrm{l})^{\mathrm{c}}$ & $-0.01(-0.08,0.05)$ & $-0.01(-0.14,0.01)$ & $0.05(-0.05,0.15)$ \\
\hline $\operatorname{ALT}(\mu \mathrm{kat} / \mathrm{l})^{\mathrm{c}}$ & $-0.32(-0.50,-0.14)$ & $-0.31(-0.50,-0.12)$ & $-0.01(-0.27,0.25)$ \\
\hline $\operatorname{AST}(\mu \mathrm{kat} / \mathrm{l})^{\mathrm{c}}$ & $-0.21(-0.34,-0.08)$ & $-0.18(-0.32,0.04)$ & $-0.03(-0.22,0.17)$ \\
\hline Fasting glucose $(\mathrm{mmol} / \mathrm{l})^{\mathrm{c}}$ & $-0.13(-0.18,-0.07)$ & $-0.07(-0.13,-0.002)$ & $-0.06(-0.15,0.03)$ \\
\hline Fasting insulin $(\mathrm{pmol} / 1)^{\mathrm{c}}$ & $-0.18(-0.35,-0.01)$ & $-0.20(-0.38,-0.02)$ & $0.02(-0.23,0.27)$ \\
\hline $\mathrm{HbA} 1 \mathrm{c}(\mathrm{mmol} / \mathrm{mol})^{\mathrm{c}}$ & $-0.07(-0.10,-0.03)$ & $-0.10(-0.14,-0.06)$ & $0.03(-0.02,0.09)$ \\
\hline Total serum amino acids $(\mathrm{mmol} / \mathrm{l})$ & $0.07(-0.09,0.24)$ & $-0.02(-0.21,0.16)$ & $0.10(-0.15,0.35)$ \\
\hline $\operatorname{BCAA}(\mu \mathrm{mol} / 1)^{\mathrm{c}}$ & $-43(-77,-9)$ & $-57(-97,-18)$ & $14(-38,67)$ \\
\hline Histidine $(\mu \mathrm{mol} / \mathrm{l})$ & $13.8(8.6,19.0)$ & $-1.6(-7.6,4.5)$ & $15.4(7.3,23.5)^{* * *}$ \\
\hline
\end{tabular}

${ }^{\text {a }}$ Mean change $(95 \% \mathrm{CI})$ within-group

${ }^{\mathrm{b}}$ Mean difference (95\% CI) of the within-group change adjusted for respective baseline values using general linear model

${ }^{\mathrm{c}}$ Not-normally distributed data have been $\log _{e}$-transformed to achieve near-normal distribution; the mean difference of the $\log _{e}$-transformed withingroup change is given

${ }^{*} p<0.05, * * * p<0.001$

ALT, alanine aminotransferase; AST, aspartate aminotransferase

did not change (Fig. 3b). Lipid oxidation was suppressed during the clamp, but did not change in either group after the intervention (Fig. 3c). Hepatic insulin sensitivity did not differ between groups before or after the intervention (Fig. 3d). Despite comparable weight loss in both groups, improvement in $R_{\mathrm{d}}$ associated with a greater weight loss in the H-RISK group, but not in the L-RISK group (Table 3).
Insulin secretion Fasting insulin levels did not change during the intervention period, while fasting blood glucose concentrations declined in both groups (Table 2). AIR tended to be lower after the H-RISK diet (mean difference $-0.05[-0.10$, $0.01] \mathrm{pmol} / 1, p=0.09$; Fig. $3 \mathrm{e}$ ) and correlated with change in $R_{\mathrm{d}}$ (Table 3), while total insulin response did not change (data not shown). DI did not change (mean difference $-0.21[-0.46$,
Fig. 1 Total cereal fibre intake (a), plasma caffeine concentration (b), $24 \mathrm{~h}$ urinary alkylresorcinol (AR) metabolite excretion (c) and intake of red meat (dark grey) or white meat (light grey) (d) in the L-RISK and H-RISK groups during the intervention period. Total whole grain fibre, $24 \mathrm{~h}$ urinary AR metabolite excretion and meat intake are expressed as means $\pm \mathrm{SD}$. Mean values have been compared between groups using Student's $t$ test for unpaired samples with Welch's correction, and not-normally distributed values were $\log _{e} e^{- \text {transformed. }}$ $* * * p<0.001$
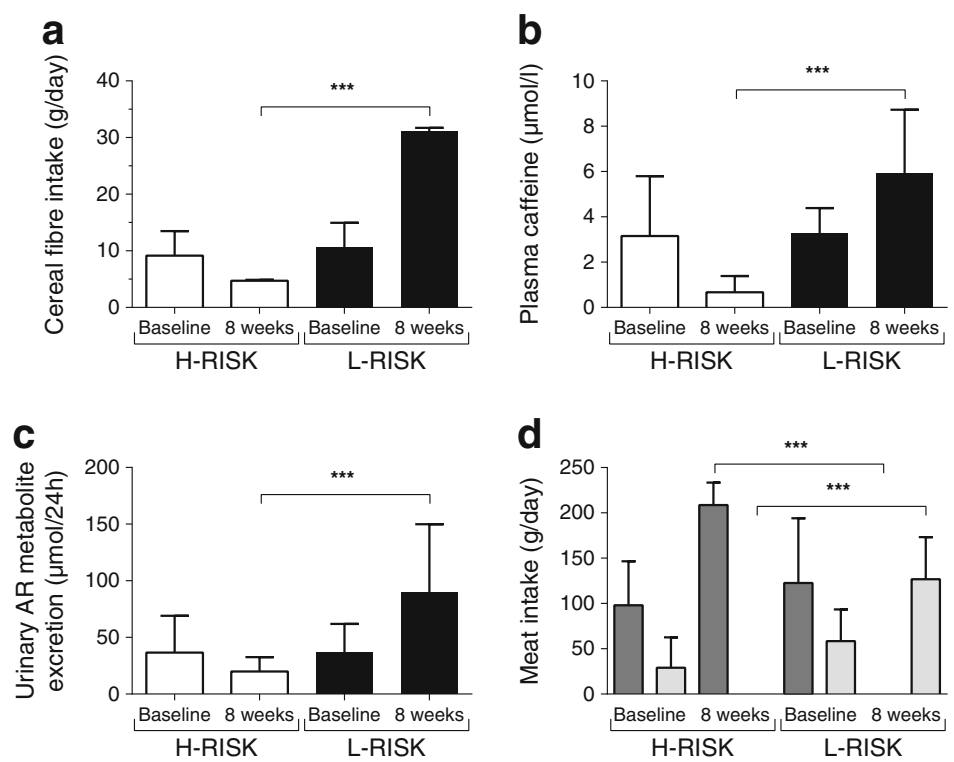
Fig. 2 Changes in body weight (a), waist circumference (b), hepatic fat content (c), SCAT (d), VAT (e) and intramyocellular fat content in M. soleus muscle (f) during run-in and intervention periods in L-RISK (black/filled circles with solid line) and $\mathrm{H}$ RISK (white/open circles with dotted line) groups. Weight and waist circumference change is given as mean per cent change \pm SD to body weight/waist circumference at screening visit (SCR). Truncal SCAT and VAT volume has been calculated from MRI images, hepatic fat content and intramyocellular fat content in soleus muscle have been assessed by ${ }^{1} \mathrm{H}$-magnetic resonance spectroscopy (MRS) and are expressed as means $\pm \mathrm{SD}$. Changes at the end of the intervention period have been compared with the end of the runin period/baseline assessment using Student's $t$ test for paired samples; not-normally distributed values were $\log _{e}$-transformed. $* p<0.05, * * p<0.01, * * * p<0.001$

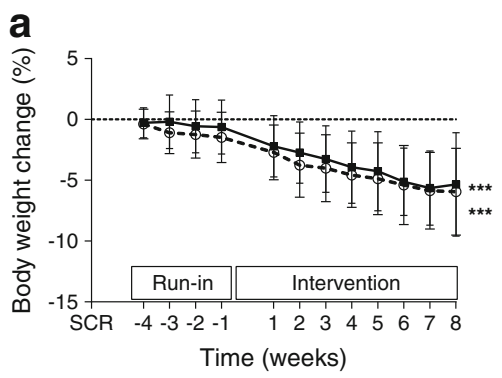

d

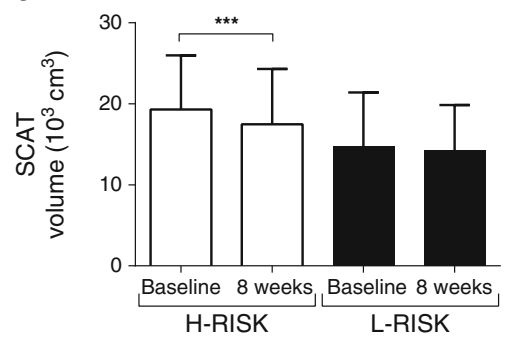

b

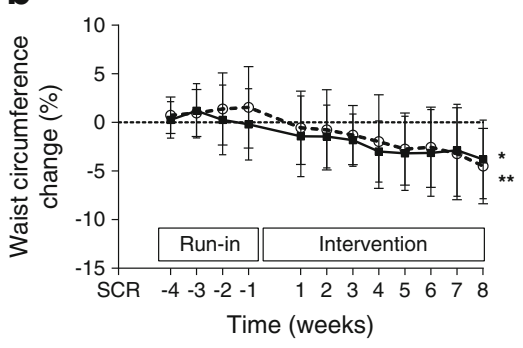

e

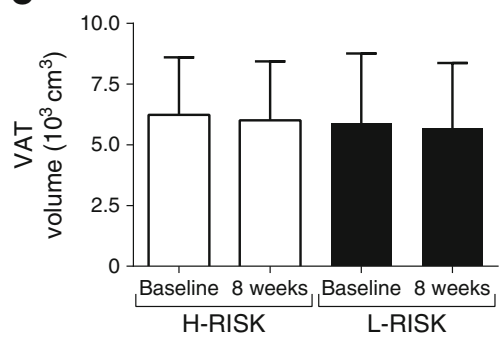

C

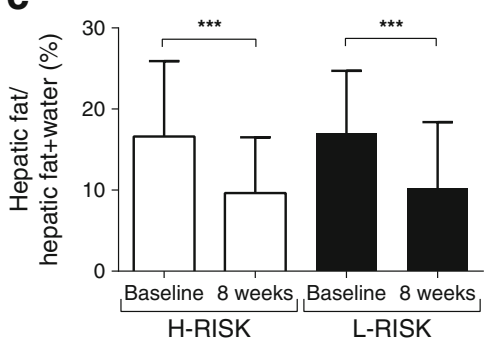

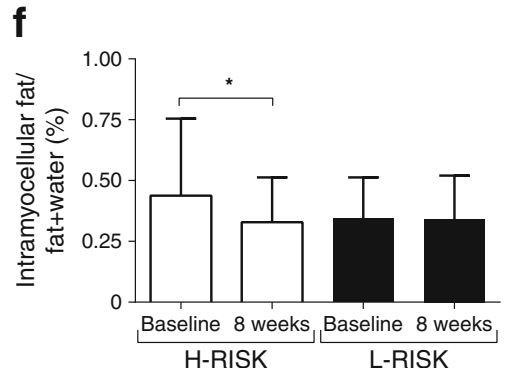

0.04]AU, $p=0.09$ ) despite a tendency towards an increase after the L-RISK diet (mean change $0.20[0.02,0.39] \mathrm{AU}$ ), but not after the H-RISK diet $(-0.01[-0.18 ; 0.16] \mathrm{AU}$; Fig. 3f).

Circulating cytokines Levels of the proinflammatory cytokine IL-18 was reduced after the L-RISK diet compared with the $\mathrm{H}-\mathrm{RISK}$ diet (mean difference $0.23[0.04,0.42] \mathrm{pg} / \mathrm{ml}$, $p<0.05$; Table 4), while all other pro- or anti-inflammatory cytokines remained unchanged. Changes in cytokine levels did not correlate with changes in insulin sensitivity or body weight (data not shown).

\section{Discussion}

This study shows that moderate energy restriction for 8 weeks irrespective of the intake of cereal fibre from wheat and rye, or intake of coffee or red meat: (1) reduces body weight, (2) increases whole-body insulin sensitivity at least partly by enhanced non-oxidative glucose utilisation, and (3) decreases HCL without affecting hepatic insulin sensitivity in type 2 diabetes. While only the L-RISK diet tended to improve subclinical inflammation, no differences between groups were observed in the reduction of body and muscle fat content or with respect to beta cell function.

Similar improvements in insulin resistance together with comparable weight loss for both diets suggests that energy restriction exclusively explains the findings. Energy restriction results in weight loss due to a reduction in body fat content and, to a lesser extent, in muscle mass. These effects associate with enhanced tissue-specific glucose uptake by increased insulinstimulated Akt2 phosphorylation [27], potentially because of a lowering of cellular lipid intermediates - possibly reflected by decreased IMCL as observed after H-RISK diet—reduced subsequent protein kinase- $\mathrm{C}$ theta $(\mathrm{PKC} \theta)$ activation and increased IRS-1 tyrosine phosphorylation [28]. Furthermore, the reduction in body weight and several fat depots correlated with insulin sensitivity only in the H-RISK group. This indicates that mechanisms other than simple weight loss may be operative.

Notably, the improved insulin resistance in the L-RISK group could occur independently of any changes in body weight or inflammatory markers, as reported for increased cereal fibre intake over $72 \mathrm{~h}$ [8]. The mechanisms responsible could be alterations to gut microbiota improving systemic inflammation independently of weight loss [29], altered digestion and/or absorption of protein subsequently affecting 
Fig. 3 Changes in whole-body insulin sensitivity (a), oxidative (light/dark grey) and nonoxidative (black/white) glucose utilisation (b), lipid oxidation rate (c), hepatic insulin sensitivity (d), AIR (e) and DI (f) before and after the intervention period. Wholebody insulin sensitivity was measured as $R_{\mathrm{d}}$ (mg [ $\mathrm{kg}$ body weight $]^{-1} \min ^{-1}$ ); glucose utilisation and lipid oxidation rates were calculated by indirect calorimetry; hepatic insulin sensitivity was measured as insulin-mediated suppression of EGP (\%); AIR (calculated during the first $10 \mathrm{~min}$ of IVGTT), and $\mathrm{DI}$ (calculated as $\mathrm{AIR} \times R_{\mathrm{d}}$ ) are given as mean \pm SD. Changes before vs end of intervention period or during fasting and clamp conditions have been compared using Student's $t$ test for paired samples; not-normally distributed values were $\log _{e^{-}}$ transformed. L-RISK group in black, H-RISK group in white. ${ }^{* *} p<0.01,{ }^{* * *} p<0.001$ a

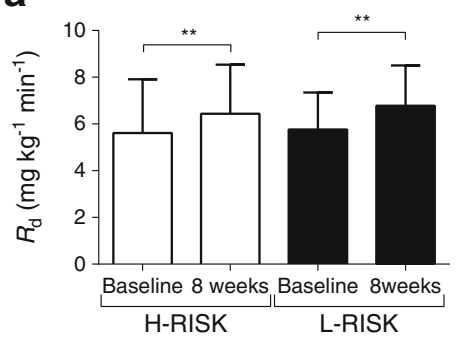

b

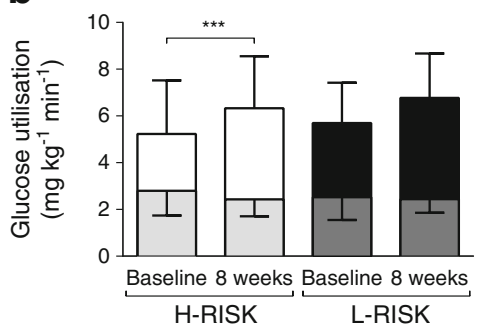

C

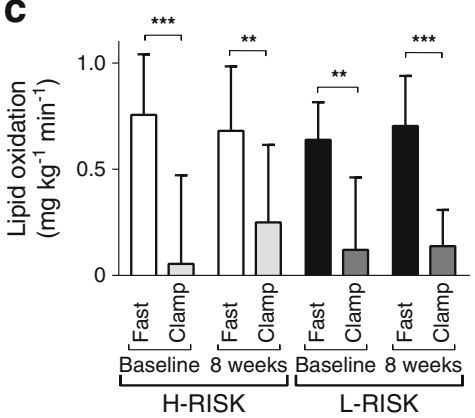

d

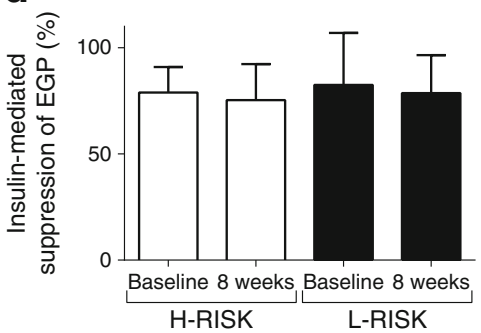

e

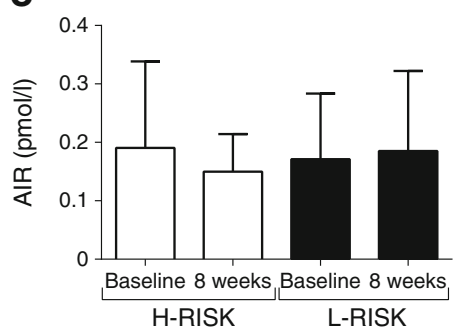

f

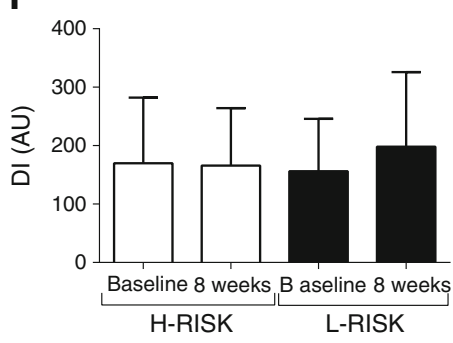

the amino acid profile [12], or altered rates of colonic fermentation with production of short-chain FA [29]. Chronic coffee

Table 3 Determinants of improvement of whole-body insulin sensitivity

\begin{tabular}{lll}
\hline & $\begin{array}{l}\text { H-RISK } \\
\text { Correlation } \\
\text { coefficient }\end{array}$ & $\begin{array}{l}\text { L-RISK } \\
\text { Correlation } \\
\text { coefficient }\end{array}$ \\
\hline Body weight change (\%) & $-0.61^{* *}$ & -0.05 \\
SCAT change (\%) & -0.23 & -0.18 \\
VAT change (\%) & -0.21 & 0.22 \\
HCL change $(\%)$ & -0.02 & 0.25 \\
a IMCL change $(\%)$ & -0.23 & 0.46 \\
Plasma caffeine change $(\mu \mathrm{mol} / \mathrm{l})$ & 0.38 & 0.40 \\
HbA1c change $(\%)$ & -0.41 & 0.06 \\
AIR change (pmol/1) & $-0.54^{*}$ & -0.22 \\
\hline
\end{tabular}

Values given are Pearson correlation coefficients $(r)$ between the absolute change of the respective variable and change in $R_{\mathrm{d}}$ during intervention; not-normally distributed parameters were analysed after $\log _{e^{-}}$ transformation

${ }^{\text {a }}$ IMCL was measured in soleus muscle

${ }^{*} p<0.05,{ }^{* *} p<0.01$ consumption has been reported to have similar effects [30]. On the other hand, coffee can impair insulin sensitivity, while liver steatosis and inflammation is reduced, despite identical and unchanged body weight in spontaneously diabetic mice [31]. Thus, L-RISK and H-RISK diets are likely to improve insulin sensitivity by different mechanisms, which may occur independently of the weight loss resulting from energy restriction.

Interestingly, we found a marked reduction in HCL after both diets, while hepatic insulin sensitivity was unaltered. Although HCL generally correlates with hepatic insulin resistance, skeletal muscle mainly accounts for improvements in whole-body insulin sensitivity [28]. The use of higher insulin doses to assess whole-body insulin sensitivity may have prevented the detection of small differences in hepatic insulin sensitivity. Nevertheless, improved hepatic insulin resistance and reduced HCL do not necessarily occur simultaneously [23]. This paradoxical finding could result from a selective insulin resistance in the liver when insulin fails to inhibit gluconeogenesis and EGP, while continuing to stimulate lipogenesis and HCL synthesis [32]. However, a very low-energy diet improves both hepatic insulin sensitivity and HCL [33], so we cannot rule out the possibility that the reduction in HCL 
Table 4 Changes in biomarkers of subclinical systemic inflammation

\begin{tabular}{llll}
\hline & H-RISK & L-RISK $^{\mathrm{a}}$ & Mean difference $^{\mathrm{b}}$ \\
\hline IL-6 $(\mathrm{pg} / \mathrm{ml})$ & $-0.04(-0.26,0.17)$ & $-0.24(-0.48,0.002)$ & $0.19(-0.13,0.52)$ \\
IL-18 $(\mathrm{pg} / \mathrm{ml})$ & $-0.01(-0.14,0.12)$ & $-0.24(-0.38,-0.19)$ & $0.23(0.04,0.42)^{*}$ \\
Adiponectin $(\mu \mathrm{g} / \mathrm{ml})$ & $-0.04(-0.20,0.12)$ & $0.13(-0.05,0.31)$ & $-0.17(-0.41,0.08)$ \\
IL-1RA $(\mathrm{pg} / \mathrm{ml})$ & $0.05(-0.13,0.22)$ & $-0.06(-0.25,0.14)$ & $0.10(-0.16,0.36)$ \\
\hline
\end{tabular}

${ }^{\text {a }}$ Mean change $(95 \% \mathrm{CI})$ within-group

${ }^{\mathrm{b}}$ Mean difference $(95 \% \mathrm{CI})$ of the within-group change adjusted for respective baseline values using general linear model

All data have been $\log _{e}$-transformed to achieve near-normal distribution and the mean difference of the $\log _{e}$-transformed within-group change is given $* p<0.05$

precedes a subsequent improvement in hepatic insulin resistance.

In line with other studies [34], this trial did not detect any effect on insulin secretion although the high cereal fibre intake during the L-RISK diet may have altered the amino acid profile $[8,12,35]$ and thereby might affect insulin secretion $[16,36]$. The observed mild reduction in the proinflammatory response may also improve insulin secretion following an L-RISK diet [37]. Furthermore, this study found no correlation between changes in insulin sensitivity and insulin secretion in either group. Thus, any adaptive effect of insulin secretion on improved insulin sensitivity was either undetectable because of the short intervention period, or counteracted by modulation of the insulin secretory response by other mechanisms that have been triggered by increased total protein and decreased fat intakes with corresponding changes in amino acids and FA [16].

This study has the strength of being a randomised controlled trial performed in well-defined, intensively phenotyped patients, which is only possible in small-scale studies. This enabled us to uncover subtle changes resulting from the interaction between dietary composition and weight loss, which would not be apparent during short-term trials in larger cohorts. Some limitations need to be considered. First, the small but significant weight loss could mask other specific effects potentially induced by the individual dietary compositions. However, this study was designed as a pretest for larger intervention trials in obese patients with type 2 diabetes, for whom weight reduction remains the primary motivation and goal of participation in the study. Moreover, detailed analysis of metabolic changes suggests different metabolic effects despite the small variation in nutrient composition. This leads to the generation of hypotheses, which need to be addressed in detail in studies using isoenergetic diets. Second, the multimodal intervention does not allow the separation of the specific effects of each dietary component, although it allowed the relevance of the net effect to be analysed in a clinical setting. Third, effects on glycaemic control, as well as the long-term effects of potentially reduced subclinical inflammation, cannot be evaluated owing to the short duration and small size of the study, although there was a tendency towards greater reduction in $\mathrm{HbA}_{1 \mathrm{c}}$. It is unclear whether a successful lifestyle intervention can reduce negative macrovascular outcomes, a major cause of mortality [7].

In conclusion, an 8 week low-energy diet reduces body weight, HCL and whole-body insulin resistance irrespective of the dietary composition in overt type 2 diabetes. Surprisingly, this short-term intervention failed to confirm previous reports of pronounced effects of high cereal wheat and rye fibre or coffee intake during phases of active weight loss, but uncovered potentially different mechanisms for both diets.

Acknowledgements We thank N. Achterath, D. Caasen-Findeisen, P. Firouzi, P. Heidkamp, S. Idler, A. Junker, I. Latta, A. Morcienitz, A. Nagel, U. Partke, B. Platzbecker, C. Preuss, D. Seeger, D. Scheibelhut, R. Schreiner and M. Schulte (all from the Institute for Clinical Diabetology, German Diabetes Center, Düsseldorf, Germany) for excellent technical assistance and taking care of the patients, as well as A. Kaija and J. Nilsson (Department of Food Science, Swedish University of Agricultural Science, Sweden) for the analyses of DHBA and DHPPA in urine samples.

Part of this work has been presented in 2013 at the 73rd Scientific Sessions of the American Diabetes Association in Chicago, and the 48th Diabetes Congress of the German Diabetes Association in Leipzig.

Funding This study was supported by the Ministry of Science and Research of the State of North Rhine-Westphalia (MIWF NRW), the German Federal Ministry of Health (BMG) and the Federal Ministry for Research (BMBF) to the Center for Diabetes Research (DZD e.V.), and the Helmholtz Alliance Imaging and Curing Environmental Metabolic Diseases (ICEMED).

Duality of interest All authors declare that there is no duality of interest associated with this manuscript.

Contribution statement MR designed the study and headed the clinical experiments. BN, LZ, AB, SK, PJN, AKF, JBG, GP, IE and RL researched the data. $\mathrm{BN}$ and $\mathrm{LZ}$ wrote the first draft of the manuscript, $\mathrm{BN}$ interpreted the statistical analysis of the data and coordinated the inclusion of specific sections as outlined. $\mathrm{AB}, \mathrm{PJN}, \mathrm{CH}, \mathrm{IE}$ and RL conducted and wrote aspects of the laboratory analyses. GP calculated indices of beta cell function and wrote the respective sections. All the authors contributed substantially to aspects of study design or the acquisition of data, contributed to drafting of the article or revised it critically for 
important intellectual content and gave final approval to the version to be published. MR is responsible for the integrity of the work as a whole.

\section{References}

1. Lindstrom J, Peltonen M, Eriksson JG et al (2008) Determinants for the effectiveness of lifestyle intervention in the Finnish Diabetes Prevention Study. Diabetes Care 31:857-862

2. Schulze MB, Hoffmann K, Boeing $H$ et al (2007) An accurate risk score based on anthropometric, dietary, and lifestyle factors to predict the development of type 2 diabetes. Diabetes Care 30:510-515

3. Noble D, Mathur R, Dent T, Meads C, Greenhalgh T (2011) Risk models and scores for type 2 diabetes: systematic review. BMJ 343 : d7163-d7194

4. Cho SS, Qi L, Fahey GC Jr, Klurfeld DM (2013) Consumption of cereal fiber, mixtures of whole grains and bran, and whole grains and risk reduction in type 2 diabetes, obesity, and cardiovascular disease. Am J Clin Nutr 98:594-619

5. Huxley R, Lee CM, Barzi F et al (2009) Coffee, decaffeinated coffee, and tea consumption in relation to incident type 2 diabetes mellitus: a systematic review with meta-analysis. Arch Intern Med 169:2053-2063

6. Pan A, Sun Q, Bernstein AM, Manson JE, Willett WC, Hu FB (2013) Changes in red meat consumption and subsequent risk of type 2 diabetes mellitus: three cohorts of US men and women. JAMA Intern Med 173:1328-1335

7. Kahn SE, Cooper ME, Del Prato S (2014) Pathophysiology and treatment of type 2 diabetes: perspectives on the past, present, and future. Lancet 383:1068-1083

8. Weickert MO, Roden M, Isken F et al (2011) Effects of supplemented isoenergetic diets differing in cereal fiber and protein content on insulin sensitivity in overweight humans. Am J Clin Nutr 94:459471

9. Andersson A, Tengblad S, Karlstrom B et al (2007) Whole-grain foods do not affect insulin sensitivity or markers of lipid peroxidation and inflammation in healthy, moderately overweight subjects. J Nutr 137:1401-1407

10. Kempf K, Herder C, Erlund I et al (2010) Effects of coffee consumption on subclinical inflammation and other risk factors for type 2 diabetes: a clinical trial. Am J Clin Nutr 91:950-957

11. Krebs JD, Parry-Strong A, Weatherall M, Carroll RW, Downie M (2012) A cross-over study of the acute effects of espresso coffee on glucose tolerance and insulin sensitivity in people with type 2 diabetes mellitus. Metabolism 61:1231-1237

12. Tremblay F, Krebs M, Dombrowski L et al (2005) Overactivation of S6 kinase 1 as a cause of human insulin resistance during increased amino acid availability. Diabetes 54:2674-2684

13. Nowotny B, Zahiragic L, Krog D et al (2013) Mechanisms underlying the onset of oral lipid-induced skeletal muscle insulin resistance in humans. Diabetes 62:2240-2248

14. Simcox JA, McClain DA (2013) Iron and diabetes risk. Cell Metab $17: 329-341$

15. Newgard CB (2012) Interplay between lipids and branched-chain amino acids in development of insulin resistance. Cell Metab 15: 606-614

16. Krebs M, Brehm A, Krssak M et al (2003) Direct and indirect effects of amino acids on hepatic glucose metabolism in humans. Diabetologia 46:917-925

17. Roden M, Stingl H, Chandramouli V et al (2000) Effects of free fatty acid elevation on postabsorptive endogenous glucose production and gluconeogenesis in humans. Diabetes 49:701-707

18. Kahl S, Nowotny B, Piepel S et al (2014) Estimates of insulin sensitivity from the intravenous-glucose-modified-clamp test depend on suppression of lipolysis in type 2 diabetes: a randomised controlled trial. Diabetologia 57:2094-2102

19. Schadewaldt P, Nowotny B, Strassburger K, Kotzka J, Roden M (2013) Indirect calorimetry in humans: a postcalorimetric evaluation procedure for correction of metabolic monitor variability. Am J Clin Nutr 97:763-773

20. Nowotny B, Nowotny PJ, Strassburger K, Roden M (2012) Precision and accuracy of blood glucose measurements using three different instruments. Diabet Med 29:260-265

21. Herder C, Bongaerts BW, Rathmann W et al (2013) Association of subclinical inflammation with polyneuropathy in the older population: KORA F4 study. Diabetes Care 36:3663-3670

22. Marklund M, Landberg R, Aman P, Kamal-Eldin A (2010) Determination of alkylresorcinol metabolites in human urine by gas chromatography-mass spectrometry. J Chromatogr B Analyt Technol Biomed Life Sci 878:888-894

23. Stefan N, Ramsauer M, Jordan P et al (2014) Inhibition of 11 betaHSD1 with RO5093151 for non-alcoholic fatty liver disease: a multicentre, randomised, double-blind, placebo-controlled trial. Lancet Diabetes Endocrinol 2:406-416

24. Laufs A, Livingstone R, Nowotny B et al (2014) Quantitative liver (31) P magnetic resonance spectroscopy at 3T on a clinical scanner. Magn Reson Med 71:1670-1675

25. Machann J, Thamer C, Stefan N et al (2010) Follow-up whole-body assessment of adipose tissue compartments during a lifestyle intervention in a large cohort at increased risk for type 2 diabetes. Radiology 257:353-363

26. Pacini G, Mari A (2003) Methods for clinical assessment of insulin sensitivity and beta-cell function. Best Pract Res Clin Endocrinol Metab 17:305-322

27. Sharma N, Arias EB, Sequea DA, Cartee GD (2012) Preventing the calorie restriction-induced increase in insulin-stimulated Akt2 phosphorylation eliminates calorie restriction's effect on glucose uptake in skeletal muscle. Biochim Biophys Acta 1822:1735-1740

28. Samuel VT, Shulman GI (2012) Mechanisms for insulin resistance: common threads and missing links. Cell 148:852-871

29. Martinez I, Lattimer JM, Hubach KL et al (2013) Gut microbiome composition is linked to whole grain-induced immunological improvements. ISME J 7:269-280

30. Cowan TE, Palmnas MS, Yang J et al (2014) Chronic coffee consumption in the diet-induced obese rat: impact on gut microbiota and serum metabolomics. J Nutr Biochem 25:489-495

31. Yamauchi R, Kobayashi M, Matsuda Y et al (2010) Coffee and caffeine ameliorate hyperglycemia, fatty liver, and inflammatory adipocytokine expression in spontaneously diabetic KK-Ay mice. J Agric Food Chem 58:5597-5603

32. Brown MS, Goldstein JL (2008) Selective versus total insulin resistance: a pathogenic paradox. Cell Metab 7:95-96

33. Lim EL, Hollingsworth KG, Aribisala BS, Chen MJ, Mathers JC, Taylor R (2011) Reversal of type 2 diabetes: normalisation of beta cell function in association with decreased pancreas and liver triacylglycerol. Diabetologia 54:2506-2514

34. Giacco R, Lappi J, Costabile G et al (2013) Effects of rye and whole wheat versus refined cereal foods on metabolic risk factors: a randomised controlled two-centre intervention study. Clin Nutr 32:941-949

35. Moazzami AA, Shrestha A, Morrison DA, Poutanen K, Mykkanen H (2014) Metabolomics reveals differences in postprandial responses to breads and fasting metabolic characteristics associated with postprandial insulin demand in postmenopausal women. J Nutr 144:807-814

36. Juntunen KS, Laaksonen DE, Poutanen KS, Niskanen LK, Mykkanen HM (2003) High-fiber rye bread and insulin secretion and sensitivity in healthy postmenopausal women. Am J Clin Nutr 77:385-391

37. Larsen CM, Faulenbach M, Vaag A et al (2007) Interleukin-1receptor antagonist in type 2 diabetes mellitus. N Engl J Med 356: $1517-1526$ 